\title{
Weak condition for generalized f-weakly Picard mappings on partial metric spaces
}

Xinchen Du, Xianjiu Huang*, Chunfang Chen

Department of Mathematics, Nanchang University, Nanchang, 330031, Jiangxi, P. R. China.

Communicated by P. Kumam

\begin{abstract}
Recently, Minak and Altun introduced the notions of multivalued weak contractions and multivalued weakly Picard operators on partial metric spaces. They also obtained two fixed point theorems with the notions of multivalued $(\delta, L)-$ weak contractions and multivalued $(\alpha, L)$-weak contractions. In this paper, we introduce the notion of generalized multivalued ( $f, \alpha, \beta)$-weak contraction on partial metric spaces. We also establish some coincidence and common fixed point theorems. Our results extend and generalize some well-known common fixed point theorems on partial metric spaces. (c)2017 All rights reserved.
\end{abstract}

Keywords: Partial metric, common fixed point, hybrid maps, weakly Picard operators.

2010 MSC: 47H10, 54H25.

\section{Introduction and preliminaries}

In recent years, many works on domain theory have been made in order to equip semantics domain with a notion of distance. In particular, Matthews [18] introduced the notion of a partial metric space as a part of the study of denotational semantics of dataflow networks. They generalized the concept of a metric space in the sense that the self-distance from a point to itself need not be equal to zero. They are useful in modeling partially defined information, which often appears in computer science. In literature [18], the contraction fixed point theorem is extended to partial metric spaces. This highlights an additional feature: the fixed point has self-distance 0 , which although trivial in metric spaces can be useful for reasoning about posets found in computer science. In the context of computer science where a computable function can also be proved to be a contraction, the partial metric extension of the contraction fixed point theorem can be used to prove that the unique fixed point, which is the programs output, will be totally computed [18]. Further applications of partial metrics to problems in theoretical computer science were discussed in [11, 12, 25, 26, 28, 29].

We start with recalling some basic definitions and lemmas on partial metric spaces. The definition of a partial metric space is given by Matthews [18].

\footnotetext{
*Corresponding author Chen)

Email addresses: xcduncu@163.com (Xinchen Du), xjhuangxwen@163.com (Xianjiu Huang), ccfygd@sina.com (Chunfang 
Definition 1.1. Let $X$ be a nonempty set, a function $p: X \times X \rightarrow R^{+}$is called a partial metric if and only if for all $x, y, z \in X$ :

$\left(p_{1}\right) x=y \Leftrightarrow p(x, x)=p(x, y)=p(y, y) ;$

$\left(p_{2}\right) p(x, x) \leqslant p(x, y)$;

$\left(p_{3}\right) p(x, y)=p(y, x)$;

$\left(p_{4}\right) p(x, z) \leqslant p(x, y)+p(y, z)-p(y, y)$.

A partial metric space is a pair $(X, p)$ such that $X$ is a nonempty set and $p$ is a partial metric on $X$. It is clear that if $p(x, y)=0$, then from $\left(p_{1}\right)$ and $\left(p_{2}\right), x=y$. But if $x=y, p(x, y)$ may not be 0 . A basic example of a partial metric space is the pair $\left(R^{+}, p\right)$, where $p(x, y)=\max \{x, y\}$ for all $x, y \in R^{+}$. Other examples of partial metric spaces which are interesting from a computational point of view may be found in [31, 32].

Each partial metric $p$ on $X$ generates a $\tau_{0}$ topology $\tau_{p}$ on $X$ which has as a base the family of open $p$-balls $\left\{B_{p}(x, \varepsilon): x \in X ; \varepsilon>0\right\}$, where $\left\{B_{p}(x, \varepsilon)=\{y \in X: p(x, y)<p(x, x)+\varepsilon\}\right.$ for all $x \in X$ and $\varepsilon>0$.

From this fact it immediately follows that a sequence $\left\{x_{n}\right\}$ in a partial metric space $(X, p)$ converges to a point $x \in X$ with respect to $\tau_{p}$ if and only if $p(x, x)=\lim _{n \rightarrow \infty} p\left(x, x_{n}\right)$. According to [18], a sequence $\left\{x_{n}\right\}$ in a partial metric space $(X, p)$ converges to a point $x \in X$ with respect to $\tau_{p^{s}}$ if and only if

$$
p(x, x)=\lim _{n \rightarrow \infty} p\left(x, x_{n}\right)=\lim _{n, m \rightarrow \infty} p\left(x_{m}, x_{n}\right) .
$$

Following [18], a sequence $\left\{x_{n}\right\}$ in a partial metric space $(X, p)$ is called a Cauchy sequence if there exists $\lim _{n, m \rightarrow \infty} p\left(x_{n}, x_{m}\right)$. A partial metric space $(X, p)$ is said to be complete if every Cauchy sequence $\left\{x_{n}\right\}$ in $X$ converges, with respect to $\mathcal{T}(p)$ to a point $x \in X$ such that $p(x, x)=\lim _{n, m \rightarrow \infty} p\left(x_{n}, x_{m}\right)$.

It is easy to see that every closed subset of a complete partial metric space is complete.

If $p$ is a partial metric on $X$, then the functions $p^{s}, p^{w}: X \times X \rightarrow R^{+}$given by

$$
p^{s}(x, y)=2 p(x, y)-p(x, x)-p(y, y)
$$

and

$$
p^{w}(x, y)=p(x, y)-\min \{p(x, x), p(y, y)\},
$$

are equivalent metric on $X$.

Lemma $1.2([18])$. Let $(\mathrm{X}, \mathrm{p})$ be a partial metric space.

(1) $\left\{x_{n}\right\}$ is a Cauchy sequence in $(X, p)$ if and only if it is a Cauchy sequence in the metric space $\left(X, p^{s}\right)$.

(2) A partial metric space $(\mathrm{X}, \mathrm{p})$ is complete if and only if the metric space $\left(\mathrm{X}, \mathrm{p}^{\mathrm{s}}\right)$ is complete. Furthermore $\lim _{n \rightarrow \infty} p^{s}\left(a, x_{n}\right)=0$ if and only if $p(a, a)=\lim _{n \rightarrow \infty} p\left(a, x_{n}\right)=\lim _{n, m \rightarrow \infty} p\left(x_{n}, x_{m}\right)$.

In [18], Matthews obtained a partial metric version of the Banach fixed point theorem. Afterward, Acar et al. [1, 2], Altun et al. [4, 5, 7, 8], Karapinar and Erhan [17], Oltra and Valero [21], Romaguera $[22,23]$ and Valero [31], gave some generalizations of the result of Matthews. Also, Ciric et al. [13], Samet et al. [27] and Shatanawi et al. [30] proved some common fixed point results in partial metric spaces. But so far all of fixed point theorems have been given for single-valued mappings. To prove Nadler's [20] fixed point theorem for multivalued maps on partial metric spaces, Aydi et al. [9] introduced the concept of partial Hausdorff distance a parallel manner to that in the Hausdorff metric in their nice paper. Then, they give some properties of partial Hausdorff distance, some important lemmas and a fundamental fixed point theorem for multivalued mappings. We can find some nice fixed point results for single-valued and multivalued maps on partial metric space in [3, 15, 19, 24, 32].

In the following, we recall the concept of partial Hausdorff distance and some properties: Let $(X, p)$ be partial metric space and $A \subseteq X$, then $A$ is said to be bounded if there exist $x_{0} \in X$ and $M \geqslant 0$ such that for all $a \in A$, we have $a \in B_{p}\left(x_{0}, M\right)$, that is, $p\left(x_{0}, a\right)<p(a, a)+M$. $A$ is closed if and only if $A=\bar{A}$, where 
$\bar{A}$ is the closure of $A$ with respect to $\tau_{p}\left(\tau_{p}\right.$ is the topology induced by $\left.p\right)$. Let $C B^{p}(X)$ be the family of all nonempty, closed and bounded subsets of $(X, p)$. For $A, B \in C B^{p}(X)$ and $x \in X$, define

$$
P(x, A)=\inf \{p(x, a): a \in A\}, \quad \delta_{p}(A, B)=\sup \{P(a, B): a \in A\},
$$

and

$$
H_{p}(A, B)=\max \left\{\delta_{p}(A, B), \delta_{p}(B, A)\right\}
$$

Lemma 1.3 ([9]). Let $(X, p)$ be a partial metric space, $A \subseteq X$ and $x \in X$. Then $x \in \bar{A}$ if and only if $P(x, A)=$ $p(x, x)$.

Proposition 1.4 ([9]). Let $(X, p)$ be a partial metric space. For any $A, B, C \in \mathrm{CB}_{p}(X)$, we have the following:

(1) $\delta_{p}(A, A)=\sup _{a \in A} p(a, a)$;

(2) $\delta_{p}(A, A) \leqslant \delta_{p}(A, B)$;

(3) $\delta_{p}(A, B)=0$ implies $A \subseteq B$;

(4) $\delta_{p}(A, B) \leqslant \delta_{p}(A, C)+\delta_{p}(C, B)-\inf _{c} \in C p(c, c)$.

Proposition $1.5([9])$. Let $(X, p)$ be a partial metric space. For any $A, B, C \in \mathrm{CB}_{p}(X)$, we have the following:

(1) $H_{p}(A, A) \leqslant H_{p}(A, B)$;

(2) $H_{p}(A, B)=H_{p}(B, A)$;

(3) $H_{p}(A, B) \leqslant H_{p}(A, C)+H_{p}(C, B)-\inf _{c} \in C p(c, c)$.

Remark 1.6. An example is given by Minak and Altun in [19] that $H_{p}(A, A)=H_{p}(A, B)=H_{p}(B, A)$, but $A \neq B$. That is, $H_{p}$ is not a partial metric on $C_{p}(X)$. Nevertheless, as shown in [9]. we have the following property:

$$
H_{p}(A, B)=0 \text { implies } A=B \text {. }
$$

Also, it is easy to see that for all $A \in \mathrm{CB}_{\mathrm{P}}(\mathrm{X})$

$$
P(a, B) \leqslant \delta_{p}(A, B) \leqslant H_{p}(A, B) .
$$

The following lemma is very important to give fixed point results for multivalued maps on partial metric space.

Lemma 1.7 ([9]). Let $(X, p)$ be a partial metric space, $A, B \in C B_{p}(X)$ and $h>1$. For any $a \in A$, there exists $\mathrm{b}=\mathrm{b}(\mathrm{a}) \in \mathrm{B}$ such that $\mathrm{p}(\mathrm{a}, \mathrm{b}) \leqslant \mathrm{hH}_{\mathrm{p}}(\mathrm{A}, \mathrm{B})$.

Lemma 1.7 can be expressed with the following version.

Lemma 1.8 ([10]). Let $(X, p)$ be a partial metric space, $A, B \in C_{p}(X)$ and $\varepsilon>0$. For any $a \in A$, there exists $b=b(a) \in B$ such that $p(a, b) \leqslant H_{p}(A, B)+\varepsilon$.

Using the partial Hausdorff distance $H_{p}$, Aydi et al. [9] proved the following fixed point theorem for multivalued mappings.

Theorem 1.9. Let $(\mathrm{X}, \mathrm{p})$ be a complete partial metric space. If $\mathrm{T}: \mathrm{X} \rightarrow \mathrm{CB}_{\mathrm{p}}(\mathrm{X})$ is a mapping such that

$$
H_{p}(T x, T y) \leqslant k p(x, y),
$$

for all $x, y \in X$, where $k \in(0,1)$, then $T$ has a fixed point.

The following theorem is a generalized version of Theorem 1.9, which is given by Altun and Minak in literature [6].

Theorem 1.10. Let $(X, p)$ be a complete partial metric space and let $\mathrm{T}: \mathrm{X} \rightarrow \mathrm{CB}_{\mathrm{p}}(\mathrm{X})$ be a multivalued map. Assume

$$
H_{p}(T x, T y) \leqslant \alpha(p(x, y)) p(x, y),
$$

for all $x, y \in X$, where $\alpha$ is an $\mathcal{M T}$-function (that is, it satisfies $\lim \sup _{s \rightarrow t^{+}} \alpha(s)<1$ for all $\mathrm{t} \in[0, \infty)$ ). Then $\mathrm{T}$ 
has a fixed point.

In literature [19], Minak and Altun generalized above theorems as follows:

Theorem 1.11. Let $(\mathrm{X}, \mathrm{p})$ be a complete partial metric space and $\mathrm{T}: \mathrm{X} \rightarrow \mathrm{CB}_{\mathrm{p}}(\mathrm{X})$ be a multivalued map such that

$$
H_{p}(T x, T y) \leqslant k p(x, y)+L P^{w}(y, T x)
$$

for all $x, y \in X$, where $k \in(0,1), L \geqslant 0$ and $\mathrm{P}^{w}(y, T x)=\inf \left\{\mathrm{p}^{w}(y, z): z \in \mathrm{T} x\right\}$. Then $\mathrm{T}$ has a fixed point.

Theorem 1.12. Let $(\mathrm{X}, \mathrm{p})$ be a complete partial metric space and let $\mathrm{T}: \mathrm{X} \rightarrow \mathrm{CB}_{\mathrm{p}}(\mathrm{X})$ be a multivalued map such that there exist an $\mathcal{M} \mathcal{T}$-function $\alpha$ and a constant $\mathrm{L} \geqslant 0$ satisfying

$$
H_{p}(T x, T y) \leqslant \alpha(p(x, y)) p(x, y)+L P^{w}(y, T x),
$$

for all $x, y \in X$. Then $T$ has a fixed point.

Recently, Huang et al. [16] gave two more general results on a partial metric space.

Theorem 1.13. Let $(X, p)$ be a partial metric space, $f: X \rightarrow X$ and $\mathrm{T}: \mathrm{X} \rightarrow \mathrm{CB}(\mathrm{X})$ be a multivalued map such that there exist two constants $\theta \in(0,1)$ and $L \geqslant 0$ satisfying

$$
H_{p}(T x, T y) \leqslant \theta p(f x, f y)+L P^{w}(f y, T x)
$$

for all $x, y \in X$ where $P^{w}(f y, T x)=\inf \left\{p^{w}(f y, z): z \in T x\right\}$ and $p^{w}$ as in (1.2). Suppose $T X \subset f X$ and $f X$ is a complete subspace of $\mathrm{X}$. Then $\mathrm{f}$ and $\mathrm{T}$ have a coincidence point $\mathrm{u} \in \mathrm{X}$. Further if $\mathrm{ffu}=\mathrm{fu}$, then $\mathrm{f}$ and $\mathrm{T}$ have $a$ common fixed point.

Theorem 1.14. Let $(X, p)$ be a partial metric space, $f: X \rightarrow X$ and $\mathrm{T}: X \rightarrow \mathrm{CB}(X)$ such that there exist an $\mathcal{M} \mathcal{T}$-function $\alpha$ and a constant $\mathrm{L} \geqslant 0$ satisfying

$$
H_{p}(T x, T y) \leqslant \alpha(p(f x, f y)) p(f x, f y)+L P^{w}(f y, T x),
$$

for all $x, y \in X$ where $P^{w}(f y, T x)=\inf \left\{p^{w}(f y, z): z \in T x\right\}$ and $p^{w}$ as in (1.2). Suppose $T X \subset f X$ and $f X$ is a complete subspace of $\mathrm{X}$. Then $\mathrm{f}$ and $\mathrm{T}$ have a coincidence point $\mathrm{u} \in \mathrm{X}$. Further if $\mathrm{ffu}=\mathrm{fu}$, then $\mathrm{f}$ and $\mathrm{T}$ have a common fixed point.

The aim of this paper is to introduce the notion of generalized multivalued $(f, \alpha, \beta)$-weak contraction on partial metric space as the parallel manner on metric space. We also establish some coincidence and common fixed point theorems with the notion of generalized multivalued $(f, \alpha, \beta)$-weak contraction on partial metric space.

\section{Main results}

As a departure, let us recall the notion of a hybrid generalized multivalued contraction mapping on partial metric spaces.

Definition 2.1 ([16]). Let $(X, p)$ be a partial metric space, $f: X \rightarrow X$ and $T: X \rightarrow \mathrm{CB}_{p}(X)$ be a multivalued operator. $T$ is said to be multivalued $f$ weakly Picard operator if and only if for each $x \in X$ and fy $\in$ $T x(y \in X)$, there exists a sequence $\left\{x_{n}\right\}$ in $X$ such that

(1) $x_{0}=x, x_{1}=y$;

(2) $f x_{n+1} \in T x_{n}$ for all $n=0,1,2, \cdots$;

(3) the sequence $\left\{f x_{n}\right\}$ converges to $f u$ where $u$ is the coincidence point of $f$ and $T$. 
Definition 2.2 ([16]). Let $\left\{x_{n}\right\}$ be a sequence in $X$ satisfying condition (1) and (2) in Definition 2.1, then the sequence $O_{f}\left(x_{0}\right)=\left\{f x_{n}: n=1,2, \cdots\right\}$ is said to be an $f$-orbit of $T$ at $x_{0}$.

Definition 2.3. Let $(X, p)$ be a partial metric space, $f: X \rightarrow X$ and $T: X \rightarrow \mathrm{CB}_{p}(X)$ be a multivalued operator. $T$ is said to be a generalized multivalued $f$-weakly contraction or a generalized multivalued $(f, \alpha, \beta)$ weak contraction if and only if there exist a function $\alpha:[0, \infty) \rightarrow[0,1)$ satisfying lim $\sup _{s \rightarrow t^{+}} \alpha(s)<1$ for every $\mathrm{t} \in[0, \infty)$ and a function $\beta:[0, \infty) \rightarrow[0,+\infty)$ such that

$$
H_{p}(T x, T y) \leqslant \alpha(p(f x, f y)) p(f x, f y)+\beta\left(P^{w}(f y, T x)\right) P^{w}(f y, T x),
$$

for all $x, y \in X$ where $P^{w}(f y, T x)=\inf \left\{p^{w}(f y, z): z \in T x\right\}$ and $p^{w}$ as in (1.2). Especially, if $\beta(x)=$ $L$ (const.) $\geqslant 0$ for every $x \in[0, \infty)$, then $T$ is said to be a generalized multivalued $(f, \alpha, L)$-weak contraction.

Remark 2.4. Due to the symmetry of $p$ and $H_{p}$, in order to check that $T$ is a multivalued $(f, \alpha, \beta)$-weak contraction on $(X, p)$, we also have to check the dual of $(2.1)$, that is to check that $T$ verifies

$$
H_{p}(T x, T y) \leqslant \alpha(p(f x, f y)) p(f x, f y)+\beta\left(P^{w}(f x, T y)\right) P^{w}(f x, T y) .
$$

Now, we give a more general result on a partial metric space. For this we need the following lemma.

Lemma $2.5([14])$. Let $\alpha:[0, \infty) \rightarrow[0,1)$ be an $\mathcal{M} \mathcal{M}$-function, then the function $\gamma:[0, \infty) \rightarrow[0,1)$ defined as $\gamma(\mathrm{t})=\frac{1+\alpha(\mathrm{t})}{2}$ is also an $\mathcal{M} \mathcal{M}$-function.

Theorem 2.6. Let $(X, p)$ be a partial metric space, $f: X \rightarrow X$ and $\mathrm{T}: \mathrm{X} \rightarrow \mathrm{CB}(\mathrm{X})$ be a multivalued $(\mathrm{f}, \alpha, \beta)$-weak contraction such that $\mathrm{TX} \subset \mathrm{fX}$. Suppose $\mathrm{fX}$ is complete. Then $\mathrm{f}$ and $\mathrm{T}$ have a coincidence point $\mathrm{u} \in \mathrm{X}$. Further, if $\mathrm{ffu}=\mathrm{fu}$, then $\mathrm{f}$ and $\mathrm{T}$ have a common fixed point.

Proof. Define a function $\gamma:[0, \infty) \rightarrow[0,1)$ as $\gamma(t)=\frac{1+\alpha(t)}{2}$, then from Lemma $2.5 \gamma(t)$ is also an $\mathcal{M} \mathcal{T}$ function. Let $x, y \in X$ be two arbitrary points with $f x \neq f y, u \in T x$ and $\varepsilon=\frac{1-\alpha(p(f x, f y))}{2} p(f x, f y)>0$ (note that since $f x \neq f y$ then $p(f x, f y)>0$ ), then from Lemma 1.8 we can find $v \in$ Ty such that $p(u, v) \leqslant$ $\mathrm{H}_{\mathrm{p}}(\mathrm{Tx}, \mathrm{Ty})+\varepsilon$. Therefore, from (2.1) we have

$$
\begin{aligned}
p(u, v) & \leqslant H_{p}(T x, T y)+\frac{1-\alpha(p(f x, f y))}{2} p(f x, f y) \\
& \leqslant \alpha(p(f x, f y)) p(f x, f y)+\beta\left(P^{w}(f y, T x)\right) P^{w}(f y, T x)+\frac{1-\alpha(p(f x, f y))}{2} p(f x, f y) \\
& =\frac{1+\alpha(p(f x, f y))}{2} p(f x, f y)+\beta\left(P^{w}(f y, T x)\right) P^{w}(f y, T x) \\
& =\gamma(p(f x, f y)) p(f x, f y)+\beta\left(P^{w}(f y, T x)\right) P^{w}(f y, T x) .
\end{aligned}
$$

Now, let $x_{0} \in X$ and $y_{0}=f x_{0}$. Since $T x_{0} \subset f X$, there exists a point $x_{1} \in X$ such that $y_{1}=f\left(x_{1}\right) \in T x_{0}$. If $y_{0}=y_{1}$, i.e., $f x_{0}=f x_{1}$, then $f x_{0} \in T x_{0}$, that is $x_{0}$ is a coincidence point of $f$ and $T$ and so the proof is complete. Let $f x_{0} \neq f x_{1}$, then from (2.2) there exists $y_{2}=f\left(x_{2}\right) \in T x_{1}$ such that

$$
\begin{aligned}
p\left(y_{1}, y_{2}\right)=p\left(f x_{1}, f x_{2}\right) & \leqslant \gamma\left(p\left(f x_{0}, f x_{1}\right)\right) p\left(f x_{0}, f x_{1}\right)+\beta\left(P^{w}\left(f x_{1}, T x_{0}\right)\right) P^{w}\left(f x_{1}, T x_{0}\right) \\
& =\gamma\left(p\left(f x_{0}, f x_{1}\right)\right) p\left(f x_{0}, f x_{1}\right) .
\end{aligned}
$$

If $y_{1}=y_{2}$, i.e., $f x_{1}=f x_{2}$, then $f x_{1} \in T x_{1}$, that is $x_{1}$ is a coincidence point of $f$ and $T$ and so the proof is complete. Let $f x_{1} \neq f x_{2}$, then from (2.2) there exists $y_{3}=f\left(x_{3}\right) \in T x_{2}$ such that

$$
\begin{aligned}
p\left(y_{2}, y_{3}\right)=p\left(f x_{2}, f x_{3}\right) & \leqslant \gamma\left(p\left(f x_{1}, f x_{2}\right)\right) p\left(f x_{1}, f x_{2}\right)+\beta\left(P^{w}\left(f x_{2}, T x_{1}\right)\right) P^{w}\left(f x_{2}, T x_{1}\right) \\
& =\gamma\left(p\left(f x_{1}, f x_{2}\right)\right) p\left(f x_{1}, f x_{2}\right) .
\end{aligned}
$$


By continuing this way, we can construct two sequences $\left\{x_{n}\right\}$ and $\left\{y_{n}\right\}$ in $X$ such that $y_{n}=f x_{n} \in T x_{n-1}$ and

$$
p\left(y_{n}, y_{n+1}\right)=p\left(f x_{n}, f x_{n+1}\right) \leqslant \gamma\left(p\left(f x_{n-1}, f x_{n}\right)\right) p\left(f x_{n-1}, f x_{n}\right),
$$

for all $n \in N$. Since $\gamma(t)<1$ for all $t \in[0, \infty)$, then $p\left(y_{n}, y_{n+1}\right)$ is a nonincreasing sequence of nonnegative real numbers. Hence $p\left(y_{n}, y_{n+1}\right)$ converges to some $\lambda \geqslant 0$. Since $\gamma(t)$ is an $\mathcal{M} \mathcal{T}$-function, then $\lim \sup \gamma(\mathrm{s})<1$ and $\gamma(\lambda)<1$. Therefore, there exist $r \in[0,1)$ and $\varepsilon>0$ such that $\gamma(\mathrm{s}) \leqslant \mathrm{r}$ for all $s \in[\lambda, \lambda+\varepsilon)$. Since $p\left(y_{n}, y_{n+1}\right) \downarrow \lambda$ we can take $k_{0} \in N$ such that $\lambda \leqslant p\left(y_{n}, y_{n+1}\right) \leqslant \lambda+\varepsilon$ for all $n \in N$ with $n \geqslant k_{0}$.

$$
p\left(y_{n+1}, y_{n+2}\right)=p\left(f x_{n+1}, f x_{n+2}\right) \leqslant \gamma\left(p\left(f x_{n}, f x_{n+1}\right)\right) p\left(f x_{n}, f x_{n+1}\right) \leqslant r p\left(f x_{n}, f x_{n+1}\right)=r p\left(y_{n}, y_{n+1}\right),
$$

for all $n \in N$ with $n \geqslant k_{0}$, then we have

$$
\begin{aligned}
\sum_{n=1}^{\infty} p\left(y_{n}, y_{n+1}\right) & \leqslant \sum_{n=1}^{k_{0}} p\left(y_{n}, y_{n+1}\right)+\sum_{n=k_{0}+1}^{\infty} p\left(y_{n}, y_{n+1}\right) \\
& =\sum_{n=1}^{k_{0}} p\left(y_{n}, y_{n+1}\right)+\sum_{n=k_{0}}^{\infty} p\left(y_{n+1}, y_{n+2}\right) \\
& \leqslant \sum_{n=1}^{k_{0}} p\left(y_{n}, y_{n+1}\right)+\sum_{n=k_{0}}^{\infty} r p\left(y_{n}, y_{n+1}\right) \\
& \leqslant \sum_{n=1}^{k_{0}} p\left(y_{n}, y_{n+1}\right)+\sum_{n=1}^{\infty} r^{n} p\left(y_{k_{0}}, y_{k_{0}+1}\right)<\infty
\end{aligned}
$$

Then for $m, n \in N$ with $m>n$, by omitting the negative term in modified triangular inequality we obtain

$$
\begin{aligned}
p\left(y_{n}, y_{m}\right) & \leqslant p\left(y_{n}, y_{n+1}\right)+p\left(y_{n+1}, y_{n+2}\right)+\cdots+p\left(y_{m-1}, y_{m}\right) \\
& =\sum_{i=n}^{m-1} p\left(y_{i}, y_{i+1}\right) \\
& \leqslant \sum_{i=n}^{\infty} p\left(y_{i}, y_{i+1}\right) \rightarrow 0 \text { as } n \rightarrow \infty
\end{aligned}
$$

Therefore, we have $\lim _{n \rightarrow \infty} p\left(y_{n}, y_{m}\right) \rightarrow 0$, that is $\left\{y_{n}=f x_{n}\right\}$ is a Cauchy sequence in $(f X, p)$. Since $(f X, p)$ is complete, $\left(f X, p^{s}\right)$ is also complete by Lemma 1.2 (2). So, there exists a point $u \in X$ such that $f x_{n} \rightarrow f u$ with respect to the metric $p^{s}$, that is $\lim _{n \rightarrow \infty} p^{s}\left(f x_{n}, f u\right)=0$.

And, by (1.1), we have

$$
p(f u, f u)=\lim _{n \rightarrow \infty} p\left(f x_{n}, f u\right)=\lim _{n, m \rightarrow \infty} p\left(f x_{m}, f x_{n}\right)=0 .
$$

Now,

$$
\begin{aligned}
P(f u, T u) & \leqslant p\left(f u, f x_{n+1}\right)+P\left(f x_{n+1}, T u\right) \\
& \leqslant p\left(f u, f x_{n+1}\right)+H_{p}\left(T x_{n}, T u\right) \\
& \leqslant p\left(f u, f x_{n+1}\right)+\alpha\left(p\left(f x_{n}, f u\right)\right) p\left(f x_{n}, f u\right)+\beta\left(P^{w}\left(f u, T x_{n}\right)\right) P^{w}\left(f u, T x_{n}\right) \\
& \leqslant p\left(f u, f x_{n+1}\right)+\alpha\left(p\left(f x_{n}, f u\right)\right) p\left(f x_{n}, f u\right)+\beta\left(P^{w}\left(f u, T x_{n}\right)\right) p^{w}\left(f u, f x_{n+1}\right) \\
& \leqslant p\left(f u, f x_{n+1}\right)+p\left(f x_{n}, f u\right)+\beta\left(P^{w}\left(f u, T x_{n}\right)\right) p^{w}\left(f u, f x_{n+1}\right) .
\end{aligned}
$$


Letting $n \rightarrow \infty$ in the above inequality we get (note that $p^{s}$ and $p^{w}$ are equivalent metrics) $P(f u, T u)=0$. Therefore, from (2.3), we obtain $P(f u, T u)=p(f u, f u)$. Thus, from Lemma 1.3, we have $f u \in T u$, since $T u$ is closed.

Let $z=f u \in T u$, then $f z=f f u=f u=z$. Using the notion of generalized multivalued $(f, \alpha, \beta)$-weak contraction, we get

$$
\begin{aligned}
H_{p}(T u, T z) & \leqslant \alpha(p(f u, f z)) p(f u, f z)+\beta\left(P^{w}(f z, T u)\right) P^{w}(f z, T u) \\
& =\alpha(p(f u, f u)) p(f u, f u)+\beta\left(P^{w}(f u, T u)\right) P^{w}(f u, T u)=0 .
\end{aligned}
$$

From $P(f z, T z)=P(f u, T z) \leqslant H_{p}(T u, T z)$, then $P(f z, T z)=0$. Therefore, from (2.3) we obtain $P(f z, T z)=$ $p(f u, f u)=p(f z, f z)$. Thus, from Lemma 1.3 we have $z=f z \in T z$, since $T z$ is closed. Thus $f$ and $T$ have a common fixed point. This completes the proof.

Remark 2.7. Let $\beta(x)=\mathrm{L}$ (const.) $\geqslant 0$, for every $x \in[0, \infty)$ in Theorem 2.6. We get Theorem 1.14.

Remark 2.8. Substituting $f=I$, the identity map on $X$ and $\beta(x)=L($ const.) $\geqslant 0$, for every $x \in[0, \infty)$ in Theorem 2.6, we get at once Theorem 1.12.

Finally, we introduce an example satisfying the hypotheses of Theorem 2.6 to support the usability of our results. In doing so, we are essentially inspired by Aydi et al. [10].

Example 2.9. Let $X=\{0,1,2,3\}$ be endowed with the partial metric $p: X \times X \rightarrow R^{+}$defined by

$$
\begin{gathered}
p(0,0)=p(1,1)=p(2,2)=0, \quad p(3,3)=\frac{1}{5}, \quad p(0,1)=p(1,0)=\frac{2}{5}, \quad p(0,2)=p(2,0)=\frac{1}{3} \\
p(1,2)=p(2,1)=\frac{2}{3}, \quad p(0,3)=p(3,0)=\frac{1}{2}, \quad p(1,3)=p(3,1)=\frac{3}{5}, \quad p(2,3)=p(3,2)=\frac{7}{10} .
\end{gathered}
$$

Also define the mappings $f: X \rightarrow X$ and $\mathrm{T}: X \rightarrow \mathrm{CB}_{\mathrm{p}}(\mathrm{X})$ by

$$
f x=\left\{\begin{array}{lc}
0 & \text { if } x \in\{0,1\}, \\
1 & \text { if } x=2, \\
2 & \text { if } x=3,
\end{array}, \quad T x=\left\{\begin{array}{l}
\{0\} \quad \text { if } x \in\{0,1,2\} \\
\{1,2\} \quad \text { if } x=3,
\end{array}\right.\right.
$$

and the $\mathcal{M} \mathcal{T}$-function $\alpha:[0, \infty) \rightarrow[0,1)$ by $\alpha(t)=\frac{6 t}{5+2 t^{2}}$ for any $t \geqslant 0$ and the function $\beta:[0, \infty) \rightarrow[0,+\infty)$ by $\beta(t)=4 t$ for any $t \geqslant 0$. Note that $T x$ is closed and bounded for all $x \in X$ under the given partial metric $p$. We shall show that (2.1) holds for all $x, y \in X$. We distinguish the following cases:

(1) If $x, y \in\{0,1,2\}$, then $H_{p}(T x, T y)=H_{p}(\{0\},\{0\})=0$ and (2.1) is satisfied obviously.

(2) If $x=0, y=3$, then

$$
\begin{aligned}
\alpha(p(f x, f y)) p(f x, f y)+\beta\left(P^{w}(f y, T x)\right) P^{w}(f y, T x) & =\alpha(p(f 0, f 3)) p(f 0, f 3)+\beta\left(P^{w}(f 3, T 0)\right) P^{w}(f 3, T 0) \\
& =\alpha(p(0,2)) p(0,2)+\beta\left(P^{w}(2,\{0\})\right) P^{w}(2,\{0\}) \\
& =\alpha\left(\frac{1}{3}\right) \frac{1}{3}+\beta\left(\frac{1}{3}\right) \frac{1}{3}=\frac{18}{47}+\frac{4}{9} \\
& =\frac{330}{423} \geqslant \frac{2}{5}=H_{p}(\{0\},\{1,2\})=H_{p}(T 0, T 3) .
\end{aligned}
$$

(3) If $x=1, y=3$, then

$$
\begin{aligned}
\alpha(p(f x, f y)) p(f x, f y)+\beta\left(P^{w}(f y, T x)\right) P^{w}(f y, T x) & =\alpha(p(f 1, f 3)) p(f 1, f 3)+\beta\left(P^{w}(f 3, T 1)\right) P^{w}(f 3, T 1) \\
& =\alpha(p(0,2)) p(0,2)+\beta\left(P^{w}(2,\{0\})\right) P^{w}(2,\{0\}) \\
& =\alpha\left(\frac{1}{3}\right) \frac{1}{3}+\beta\left(\frac{1}{3}\right) \frac{1}{3}=\frac{18}{47}+\frac{4}{9} \\
& =\frac{330}{423} \geqslant \frac{2}{5}=H_{p}(\{0\},\{1,2\})=H_{p}(T 1, T 3) .
\end{aligned}
$$


(4) If $x=2, y=3$, then

$$
\begin{aligned}
\alpha(p(f x, f y)) p(f x, f y)+\beta\left(P^{w}(f y, T x)\right) P^{w}(f y, T x) & =\alpha(p(f 2, f 3)) p(f 2, f 3)+\beta\left(P^{w}(f 3, T 2)\right) P^{w}(f 3, T 2) \\
& =\alpha(p(1,2)) p(1,2)+\beta\left(P^{w}(2,\{0\})\right) P^{w}(2,\{0\}) \\
& =\alpha\left(\frac{2}{3}\right) \frac{2}{3}+\beta\left(\frac{1}{3}\right) \frac{1}{3}=\frac{24}{53}+\frac{4}{9} \\
& =\frac{428}{477} \geqslant \frac{2}{5}=H_{p}(\{0\},\{1,2\})=H_{p}(T 2, T 3) .
\end{aligned}
$$

(5) If $x=3, y=0$, then

$$
\begin{aligned}
\alpha(p(f x, f y)) p(f x, f y)+\beta\left(P^{w}(f y, T x)\right) P^{w}(f y, T x) & =\alpha(p(f 3, f 0)) p(f 3, f 0)+\beta\left(P^{w}(f 0, T 3)\right) P^{w}(f 0, T 3) \\
& =\alpha(p(2,0)) p(2,0)+\beta\left(P^{w}(0,\{1,2\})\right) P^{w}(0,\{1,2\}) \\
& =\alpha\left(\frac{1}{3}\right) \frac{1}{3}+\beta\left(\frac{1}{3}\right) \frac{1}{3}=\frac{18}{47}+\frac{4}{9} \\
& =\frac{330}{423} \geqslant \frac{2}{5}=H_{p}(\{1,2\},\{0\})=H_{p}(T 3, T 0) .
\end{aligned}
$$

(6) If $x=3, y=1$, then

$$
\begin{aligned}
\alpha(p(f x, f y)) p(f x, f y)+\beta\left(P^{w}(f y, T x)\right) P^{w}(f y, T x) & =\alpha(p(f 3, f 1)) p(f 3, f 1)+\beta\left(P^{w}(f 1, T 3)\right) P^{w}(f 1, T 3) \\
& =\alpha(p(2,0)) p(2,0)+\beta\left(P^{w}(0,\{1,2\})\right) P^{w}(0,\{1,2\}) \\
& =\alpha\left(\frac{1}{3}\right) \frac{1}{3}+\beta\left(\frac{1}{3}\right) \frac{1}{3}=\frac{18}{47}+\frac{4}{9} \\
& =\frac{330}{423} \geqslant \frac{2}{5}=H_{p}(\{1,2\},\{0\})=H_{p}(T 3, T 1) .
\end{aligned}
$$

(7) If $x=3, y=2$, then

$$
\begin{aligned}
\alpha(p(f x, f y)) p(f x, f y)+\beta\left(P^{w}(f y, T x)\right) P^{w}(f y, T x) & =\alpha(p(f 3, f 2)) p(f 3, f 2)+\beta\left(P^{w}(f 2, T 3)\right) P^{w}(f 2, T 3) \\
& =\alpha(p(2,1)) p(2,1)+\beta\left(P^{w}(1,\{1,2\})\right) P^{w}(1,\{1,2\}) \\
& =\alpha\left(\frac{2}{3}\right) \frac{2}{3}+0=\frac{24}{53} \\
& \geqslant \frac{2}{5}=H_{p}(\{1,2\},\{0\})=H_{p}(T 3, T 2) .
\end{aligned}
$$

(8) If $x=y=3$, then $H_{p}(T x, T y)=H_{p}(\{1,2\},\{1,2\})=0$ and (2.1) is satisfied obviously. Thus, all the conditions of Theorem 2.6 are satisfied and $x=0$ is a common fixed point of $f$ and $T$ in $X$.

\section{Acknowledgment}

The authors thank the editor and the referees for their valuable comments and suggestions. This research has been supported by the National Natural Science Foundation of China $(11461043,11661053)$ and supported partly by the Provincial Natural Science Foundation of Jiangxi, China (20161BAB201009) and the Science and Technology Project of Educational Commission of Jiangxi Province, China (150013).

\section{References}

[1] Ö. Acar, I. Altun, Some generalizations of Caristi type fixed point theorem on partial metric spaces, Filomat, 26 (2012), 833-837. 1

[2] Ö. Acar, I. Altun, S. Romaguera, Caristi's type mappings on complete partial metric spaces, Fixed Point Theory, 14 (2013), 3-9.1 
[3] Ö. Acar, V. Berinde, I. Altun, Fixed point theorems for iri-type strong almost contractions on partial metric spaces, J. Fixed Point Theory Appl., 12 (2012), 247-259. 1

[4] I. Altun, Ö. Acar, Fixed point theorems for weak contractions in the sense of Berinde on partial metric spaces, Topology Appl., 159 (2012), 2642-2648. 1

[5] I. Altun, A. Erduran, Fixed point theorems for monotone mappings on partial metric spaces, Fixed Point Theory Appl., 2011 (2011), 10 pages. 1

[6] I. Altun, G. Minak, Mizoguchi-Takahashi type fixed point theorem on partial metric spaces, J. Adv. Math. Stud., 7 (2014), 80-88. 1

[7] I. Altun, S. Romaguera, Characterizations of partial metric completeness in terms of weakly contractive mappings having fixed point, Appl. Anal. Discrete Math., 6 (2012), 247-256. 1

[8] I. Altun, F. Sola, H. Simsek, Generalized contractions on partial metric spaces, Topology Appl., 157 (2010), $2778-2785$. 1

[9] H. Aydi, M. Abbas, C. Vetro, Partial Hausdorff metric and Nadler's fixed point theorem on partial metric spaces, Topology Appl., 159 (2012), 3234-3242. 1, 1.3, 1.4, 1.5, 1.6, 1.7, 1

[10] H. Aydi, M. Abbas, C. Vetro, Common fixed points for multivalued generalized contractions on partial metric spaces, Rev. R. Acad. Cienc. Exactas Fs. Nat. Ser. A Math. RACSAM, 108 (2014), 483-501. 1.8, 2

[11] M. A. Bukatin, J. S. Scott, Towards computing distances between programs via Scott domains, Logical foundations of computer science, Yaroslavl, (1997), Lecture Notes in Comput. Sci., Springer, Berlin, 1234 (1997), 33-43. 1

[12] M. A. Bukatin, S. Y. Shorina, Partial metrics and co-continuous valuations, Foundations of software science and computation structures, Lisbon, (1998), Lecture Notes in Comput. Sci., Springer, Berlin, 1378 (1998), 125-139. 1

[13] L. Ćirić, B. Samet, H. Aydi, C. Vetro, Common fixed points of generalized contractions on partial metric spaces and an application, Appl. Math. Comput., 218 (2011), 2398-2406. 1

[14] W.-S. Du, Some new results and generalizations in metric fixed point theory, Nonlinear Anal., 73 (2010), 1439-1446. 2.5

[15] R. H. Haghi, S. Rezapour, N. Shahzad, Be careful on partial metric fixed point results, Topology Appl., 160 (2013), 450-454. 1

[16] X.-J. Huang, Y.-Y. Li, C.-X. Zhu, Multivalued f-weakly Picard mappings on partial metric spaces. J. Nonlinear Sci. Appl., 8 (2015) 1234-1244. 1, 2.1, 2.2

[17] E. Karapınar, İ. M. Erhan, Fixed point theorems for operators on partial metric spaces, Appl. Math. Lett., 24 (2011), 1894-1899. 1

[18] S. G. Matthews, Partial metric topology, Papers on general topology and applications, Flushing, NY, (1992), Ann. New York Acad. Sci., New York Acad. Sci., New York, 728 (1994), 183-197. 1, 1, 1, 1.2, 1

[19] G. Minak, I. Altun, Multivalued weakly Picard operators on partial metric spaces, Nonlinear Funct. Anal. Appl., 19 (2014), 45-59. 1, 1.6, 1

[20] S. B. Nadler, Jr., Multi-valued contraction mappings, Pacific J. Math., 30 (1969), 475-488. 1

[21] S. Oltra, O. Valero, Banach's fixed point theorem for partial metric spaces, Rend. Istit. Mat. Univ. Trieste, 36 (2004), 17-26. 1

[22] S. Romaguera, Matkowski's type theorems for generalized contractions on (ordered) partial metric spaces, Appl. Gen. Topol., 12 (2011), 213-220. 1

[23] S. Romaguera, Fixed point theorems for generalized contractions on partial metric spaces, Topology Appl., 159 (2012), 194-199. 1

[24] S. Romaguera, On Nadler's fixed point theorem for partial metric mpaces, Math. Sci. Appl. E-Notes, 1 (2013), 1-8. 1

[25] S. Romaguera, M. Schellekens, Duality and quasi-normability for complexity spaces, Appl. Gen. Topol., 3 (2002), 91-112. 1

[26] S. Romaguera, M. Schellekens, Weightable quasi-metric semigroups and semilattices, Electron. Notes Theor. Comput. Sci., 40 (2003), 347-358. 1

[27] B. Samet, M. Rajović, R. Lazović, R. Stojiljković, Common fixed-point results for nonlinear contractions in ordered partial metric spaces, Fixed Point Theory Appl., 2011 (2011), 14 pages. 1

[28] M. P. Schellekens, A characterization of partial metrizability: domains are quantifiable, Topology in computer science, Schloß Dagstuhl, (2000). Theoret. Comput. Sci., 305 (2003), 409-432. 1

[29] M. P. Schellekens, The correspondence between partial metrics and semivaluations, Theoret. Comput. Sci., 315 (2004), 135-149. 1

[30] W. Shatanawi, B. Samet, M. Abbas, Coupled fixed point theorems for mixed monotone mappings in ordered partial metric spaces, Math. Comput. Modelling, 55 (2012), 680-687. 1

[31] O. Valero, On Banach fixed point theorems for partial metric spaces, Appl. Gen. Topol., 6 (2005), 229-240. 1, 1

[32] X. Wen, X.-J. Huang, Common fixed point theorem under contractions in partial metric spaces, J. Comput. Anal. Appl., 13 (2011), 583-589. 1, 1 